\title{
Relationship Between Intelligence and Criterion Task Set Performance 1
}

\author{
Kirby Gilliland, Robert Schlegel, Sharon Dannels, and Scott Mills \\ University of Oklahoma \\ Norman, Oklahoma
}

\begin{abstract}
Intelligence has been shown to be a mediating factor in the performance of many tasks. The purpose of this study is to examine the relationship between Wechsler Adult Intelligence Scale-Revised (WAIS-R) scores and performance on a multi-task performance battery, the USAF Criterion Task Set (CTS). Performance scores for high and low WAIS-R groups $(\mathrm{N}=26$ /group) were compared across each task component of the CTS. Results of this study indicate that there is a fairly strong relationship between intelligence, as measured by WAIS-R, and performance on the CTS central processing tasks. Subjects scoring high on the WAIS-R are likely to be more accurate and faster in their responses than subjects who scored low on the WAIS-R. Verbal and performance subscales of the WAIS-R did not appear to mediate task performance differentially. In general, WAIS-R performance does not seem to be related to perceptual input tasks or motor/output tasks of the CTS battery.
\end{abstract}

\section{INTRODUCTION}

There are many individual difference variables that are known to relate to the manner in which people process information or to the processes related directly to performance capability. As operator environments become increasingly complex, these individual differences become more important in the determination and prediction of operator performance.

Intelligence is one of the many variables that is known to be related in some manner to human performance. While the theoretical nature of general intelligence remains controversial, this dimension has been shown to be a mediating factor in the performance of many tasks. For example, it has been shown that intelligence is correlated negatively with inspection time, reaction time, and speed of performance in the Sternberg task. Intelligence has also been shown to be positively correlated with accuracy measures of spatial visualization and numerous measures of verbal ability. (See reviews by Eysenck, 1987; Hunt, 1978; Jensen, 1982; Nettelbeck, 1987; and Vernon; 1987.) The purpose of the present study is to examine the relationship between performance on the Wechsler Adult Intelligence Scale-Revised (WAIS-R) and performance on a multi-task performance battery.

\section{METHOD}

\section{Subjects}

Subjects in the study were subsamples from a total of 95 , male volunteers who participated in a large standardization study of the Criterion Task Set (as described below). These subjects varied in age from 19 to 34 years, had no significant hearing or vision impairments, and were not taking any medication. The data analyzed for this investigation were based on subjects in the standardization study (approximately 26 per group) who fell in the upper and lower $27 \%$ of the distribution of those completing the WAIS-R (see Cox, 1959).

\section{Procedure}

In this comparison of intelligence and task performance, it was important to select a task battery that would assess a wide range of cognitive and perceptual motor abilities. The U.S. Air Force Criterion Task Set (CTS; Shingledecker, 1984) represents one effort to develop a comprehensive, standardized workload assessment battery based on current theoretical models of human information processing. The CTS battery is composed of nine tasks with each task designed to assess one of three primary stages of processing (perceptual/input, central processing, and motor/output). The CTS battery contains the following tasks:

\section{Perceptual/Input}

-Probability Monitoring Task

\section{Central Processing}

-Memory Search Task

-Continuous Recall Task

- Linguistic Processing Task

-Grammatical Reasoning Task

-Mathematical Processing Task

-Spatial Processing Task

\section{Motor/Output}

- Unstable Tracking Task -Interval Production Task 
The CTS battery provides three workload (difficulty) levels for all of the tasks, except the Interval Production (tapping) task, which has only one difficulty level. Thus, the CTS battery includes 25 separate tasks.

The CTS has been applied as a test instrument to evaluate the relative sensitivity, reliability, and intrusiveness of a variety of available workload measures (Shingledecker, Acton, \& Crabtree, 1983). It has also been used as a performance assessment battery to evaluate the effects of various stressors on individual components of the information processing system.

A large-scale standardization study forming a comprehensive data base including CTS performance data, subjective workload assessments, and numerous measures of individual difference variables has recently been completed (see Schlegel \& Gilliland, 1988 for details of the testing protocol). Briefly, performance data on the CTS were collected for 95 male subjects who performed all 25 CTS tasks, each day, for 9 days. Five days (trials) were allowed for training, two days for baseline testing, and two days for testing under various stressors. This paper will summarize the relationship between WAIS-R scales for selected subjects and CTS performance data from the first day of baseline testing.

\section{RESULTS}

Scores on the CTS performance tasks were grouped into the following categories: a) percent correct measures for the six central processing tasks, b) mean response time measures for the six central processing tasks, c) various dependent measures for the two motor output tasks, and d) various dependent measures for the single perceptual input task. Multivariate analysis of variance tests were performed on these groupings of dependent measures followed, where appropriate, by univariate and multiple comparison (Ryan) tests.

The MANOVA results revealed a significant main effect for WAIS-R group, $F(6,45)=5.43, p<$ .0003 , and a WAIS-R by Task Difficulty Level interaction, $F(12,190)=2.52, p<.004$, for the percent correct measure on the six central processing tasks of the CTS battery. Univariate analyses revealed significant main effect differences for Linguistic Processing ,Grammatical Reasoning, Spatial Processing, and Continuous Recall tasks and significant interaction effects for Linguistic Processing, Grammatical Reasoning, Spatial Processing, and Continuous Recall tasks. These analyses revealed that subjects in the high WAIS-R group had a higher percentage correct than subjects in the low WAIS-R group. In all cases, the significant interactions were due to a much more rapid decline in performance across the workload levels for the low WAIS-R group as compared to the high WAIS-R group.

MANOVA analyses were also performed on mean response time for the six CTS central processing tasks. Again, these analyses yielded significant differences for the WAIS- $R$ main effect, $F(6,45)=2.97, p<.015$, and the WAIS-R by Task
Difficulty Level interaction, $F(12,190)=2.57, p<$ .004 . The univariate analyses confirmed that the high WAIS-R group had significantly lower mean response times on the Grammatical Reasoning, Spatial Processing, Math Processing, Memory Search, and Linguistic Processing tasks as compared to the low WAIS-R group. Significant interactions were due primarily to a more rapid increase in response time across the task difficulty levels on the Continuous Recall and Math Processing tasks for the low WAIS-R group, as compared to the high WAISR group.

MANOVA analysis yielded no significant differences for any dependent measure related to either of the motor/output tasks (Unstable Tracking and Interval Production), or the perceptual/input (Probability Monitoring) task.

Analysis of Verbal and Performance subscales of the WAIS-R revealed patterns of differences that were similar to those found for the WAIS-R overall score. No marked differences were found that would suggest either of these subscales markedly mediated performance differentially. Again, no significant differences were found in the subscale analyses for any dependent measure for the motor/output tasks or for the perceptual/input task.

\section{DISCUSSION}

Results of this study indicate that there is a fairly strong relationship between intelligence, as measured by WAIS-R, and performance on the CTS central processing tasks. Subjects scoring high on the WAIS-R are more likely to be both more accurate and faster in their responses on the Linguistic Processing, Grammatical Reasoning, and Spatial Processing tasks than subjects who scored low on the WAIS-R. These results are in agreement with much of the existing literature (e.g., Hunt, 1978; Jensen, 1982; Ozer,1987; and Vernon, 1987).

High WAIS-R scorers were also more accurate (but equal in speed) in responding to the Continuous Recall task, and quicker (but equal in accuracy) in responding to the Math Processing and Memory Search tasks, as compared to low WAIS-R scorers.. Performance on the WAIS-R was not found to be related to dependent measures on the Probability Monitoring task--a finding somewhat at odds with existing literature on speed of information processing and inspection time (cf., Eysenck, 1987 and Nettelbeck, 1987). And, WAIS-R performance did not seem to be related to either of the motor/output tasks of the CTS battery.

This investigation offered the unique opportunity to examine the relationship between WAIS-R score and performance on a wide range of performance tasks for the same subjects. The results of this study suggested that WAIS-R performance was highly related to a number of speed and accuracy measures on the CTS central processing tasks. The results of this study also suggested that WAIS-R score was not related to dependent measures on the input/perceptual task 
or either of the tasks emphasizing motor/output functions.

1 This research was sponsored in part by the Workload and Ergonomics Branch of the Armstrong Aerospace Medical Research Laboratory, United States Air Force, under Contract F33615-85-D-0514 through the Southeastern Center for Electrical Engineering Education (SCEEEHER/86-9). The United States Government is authorized to reproduce and distribute reprints for government purposes notwithstanding any copyright notation hereon. The authors wish to thank Gary Reid for his interest and support in the completion of this project.

\section{REFERENCES}

Cox, D. (1957). Note on grouping. Journal of the American Statistical Association, 52, 543-547.

Eysenck, H.J. (1987). Speed of information processing, reaction time, and the theory of intelligence. In P. A. Vernon (Ed.) Speed of information processing and intelligence. Norwood, New Jersey: Ablex Publishing Corp.

Hunt, E. (1978). The next word on verbal ability. In P. A. Vernon (Ed.) Speed of informationprocessing and intelligence. Norwood, New Jersey: Ablex Publishing Corp.

Jensen, A. R. (1982). Reaction time and psychometric g. In H.J. Eysenck (Ed.) $A$ model for intelligence. New York, New York: SpringerVerlag.
Nettelbeck, T. (1987). Inspection time and intelligence. In P. A. Vernon (Ed.) Speed of information-processing and intelligence. Norwood, New Jersey: Ablex Publishing Corp.

Ozer, D. (1987). Personality, intelligence, and spatial visualization: Correlates of Mental Rotations Test performance. Journal of Personality and Social Psychology, 53, 129-134.

Schlegel, R., \& Gilliland, K. (1988). Evaluation of the Criterion Task Set. (Technical Report AAMRL-TR-88-in press) Wright-Patterson AFB, OH: Armstrong Aerospace Medical Research Laboratory.

Shingledecker, C. A. (1984). A task battery for applied human performance assessment research. (Technical Report AFAMRL-TR-84071). Wright-Patterson AFB, OH: Armstrong Aerospace Medical Research Laboratory.

Shingledecker, C. A., Acton, W., \& Crabtree, M. (1983). Development and application of a Criterion Task Set for workload metric evaluation. Second Aerospace Behavioral Engineering Technology Conference Proceedings, Society for Automotive Engineers, 43-49.

Vernon, P. A. (1987). New developments in reaction time research. In P. A. Vernon (Ed.) Speed of information-processing and intelligence. Norwood, New Jersey: Ablex Publishing Corp. 